\title{
Akademische Nachrufe als Datensorte für die Geschichte der Soziologie
}

\author{
Julian Hamann, Forum Internationale Wissenschaft, University of Bonn
}

\begin{abstract}
Der Beitrag behandelt in Fachzeitschriften veröffentliche Nachrufe als Datenmaterial, die das wissenschaftliche Personal für die (soziologische) Fachgeschichte sichtbar macht. Nachrufe eröffnen einen spezifischen Blick auf die Konstruktion wissenschaftlicher Biografien und Lebensleistungen. Die besonderen Hintergründe der Produktion und Rezeption dieses Genres können produktiv genutzt werden, wenn die Analyse reflektiert, dass es sich hier nicht um dokumentarische Darstellungen von Biografien handelt, sondern um ihre Narration und Bewertung. Die diesen Vorgängen zugrunde liegenden Regeln illustrieren die ansonsten weitgehend implizit bleibenden Kriterien, die an wissenschaftliche Biografien und Lebensleistungen angelegt werden.
\end{abstract}

\section{Einleitung}

Sobald sich fachgeschichtliche Darstellungen dem wissenschaftlichen Personal zuwenden, stoßen sie auf eine Schwierigkeit: Die Wissenschaft ist geradezu programmatisch von ihrem Personal getrennt. Seit Beginn der modernen Wissenschaft sollen auf diese Weise die Rationalität und die Gültigkeit wissenschaftlicher Aussagen vom sozialen Stand einer Person abgekoppelt werden. Das führt dazu, dass Wissenschaftlerinnen ${ }^{1}$ von sich selbst zu schweigen und sich hinter ihren Aussagen möglichst unsichtbar zu machen haben (Kohli 1981). Mit ,Autor' oder auch ,Verfasser' existieren zwar diskursive Sprecherpositionen, die Kohärenz und Orientierung versprechen sowie für die Wahrheit einer Aussage bürgen sollen (Foucault 1977; Etzemüller 2013). Diskursive Sprecherpositionen sorgen jedoch gerade nicht dafür, dass der Jemand, dem die Aussage zugeschrieben wird, als Person sichtbar wird. In diesem Beitrag will ich mit akademischen Nachrufen ein empirisches Datenmaterial beschreiben, das einen Zugriff auf das wissenschaftliche Personal erlaubt.

Die Norm der Selbstbeschweigung ist von der Wissenschaftssoziologie vor allem in ihren Anfängen nicht etwa dekonstruiert, sondern reifiziert worden. Für Max Weber (1922, S. 533) hat es der modernen Wissenschaft nicht um die Person, sondern um die Sache zu gehen; er schwört die Soziologie bereits früh darauf ein, dass „,Persönlichkeit' auf wissenschaftlichem Gebiet" nur der habe, „der rein der Sache dient". Robert K. Merton (1973, S. 276) beschreibt das programmatische Desinteresse an der Person sogar als grundlegendes Element „of science itself“. In der Praxis führt die Auslassung personaler Bezüge dazu, dass zum Teil noch heute bei der Formulierung wissenschaftlicher Texte das ,Ich`vermieden und auf selbstreferenzielle Passivkonstruktionen zurückgegriffen wird 
(Gusfield 1976; Emihovich 1995 - wie bereits erwähnt bedeutet das freilich nicht, dass akademische Texte keine engagierten Sprecherpositionen aufweisen, vgl. Hyland 2004).

Mittlerweile kann die (Wissenschafts-)Soziologie die wissenschaftliche Selbstbeschweigung als einen recht effektiven Zensurmechanismus wissenschaftlicher Diskurse dekonstruieren (Foucault 1991). Bei der demonstrativ zur Schau getragenen Interessenlosigkeit an der Person handelt es sich selbst um ein Interesse des wissenschaftlichen Feldes. Die Selbstbeschweigung ist somit Teil der feldinternen Illusio, ihre Rekonstruktion wird zu einem wichtigen Beitrag zur Analyse des wissenschaftlichen Feldes und seiner Agenten (Bourdieu 2004). Damit ist zwar ein Erklärungsansatz für die Unsichtbarmachung und Selbstbeschweigung des wissenschaftlichen Personals geliefert, das forschungspraktische Problem ist damit aber weder für die Wissenschaftsforschung im Allgemeinen noch für die soziologische Fachgeschichte im Besonderen gelöst. Wie können also wissenschaftliche Personen sichtbar gemacht werden?

In der Tat existiert eine ganze Reihe von Datensorten, die je unterschiedliche empirische Zugriffe auf das wissenschaftliche Personal erlauben. Mein Beitrag wird in Fachzeitschriften veröffentlichte Nachrufe als Datenmaterial vorstellen, in dem das Schweigen über das wissenschaftliche Personal gebrochen wird, um wissenschaftliche Biografien und Lebensleistungen zu konstruieren und zu bewerten. Der Forschungsstand zu Nachrufen deutet das Potenzial des Datenmaterials an, zeigt aber auch, dass die systematische Beschäftigung mit Nachrufen bisher für die Wissenschaftsgeschichte und erst recht für die Wissenschaftssoziologie ein Desiderat ist (2). Dies ist überraschend, stellen sich Nachrufe bei näherer Betrachtung doch als reichhaltige Quelle für die Darstellung und Bewertung wissenschaftlicher Biografien und Lebensleistungen heraus (3). Die spezifischen Charakteristika von Nachrufen zeigen sich besonders deutlich im Vergleich mit ähnlichen Datensorten wie Biografien und Curricula Vitae (4).

\section{Forschungsstand zu Nachrufen}

Weil weitere Datensorten zum wissenschaftlichen Personal in anderen Beiträgen dieses Handbuchs diskutiert werden (vgl. z. B. die Beiträge zu Biografien oder Korrespondenzen von Jennifer Platt und Fabian Link in diesem Band) und in meinem Beitrag ein eigener vergleichender Abschnitt folgt, kann sich der Forschungsstand auf Studien zu Nachrufen konzentrieren. Dabei stellen in Zeitungen veröffentlichte Nachrufe auf Personen des öffentlichen Lebens eine wichtige Ergänzung zu akademischen Nachrufen in Fachzeitschriften dar (zu den Unterschieden zwischen akademischen und Zeitungsnachrufen siehe den vergleichenden Abschn. $\underline{4}$ dieses Beitrags).

Grundsätzlich kann die Forschung zu Zeitungsnachrufen in eher sozialstrukturanalytische und in eher sozialkonstruktivistische Arbeiten unterteilt werden. Sozialstrukturanalytische Perspektiven interessieren sich zum Beispiel dafür, für Vertreter welcher sozialen Gruppen überhaupt Nachrufe verfasst werden. Nachrufe erscheinen dann als kollektives Gedächtnis (Fowler 2007), dessen Selektivität kritisch beleuchtet wird. Die Annahme ist, dass durch Nachrufe tendenziell jene Personen öffentlich legitimiert werden, die ohnehin schon eine sozial und materiell privilegierte Position innehaben bzw. vor ihrem Tod innehatten (Fowler 2005b). Für die jüngere Zeit ist eine zunehmende Inklusion, alternativer Eliten' wie beispielsweise popkultureller Figuren festgestellt worden (Fowler 2005a). Der Anteil von in Nachrufen erinnerten Frauen steigt ebenfalls an (Eid 2002; Fowler und Bielsa 2007). 
Sozialkonstruktivistische Perspektiven fragen danach, welche Identitäten in Nachrufen symbolisiert und repräsentiert werden (Bonsu 2007). Gleichen sich weibliche den männlich kodierten Führungspersönlichkeiten an, weil Frauen zunehmend ebenfalls als engagiert und professionell beschrieben werden (Rodler et al. 2001)? Konstruktivistische Studien setzen sich auch in grundsätzlicherer Form damit auseinander, wie Nachrufe Lebensläufe erzählen (Bytheway und Johnson 1996) und inwiefern sich in ihnen die Außenwelt widerspiegelt, wenn beispielsweise Organisationen (Long 1987) oder öffentliche Großereignisse wie Kriege (Taussig 2016) in die Erzählung eingeflochten werden.

Lassen wir die Differenzen zwischen Zeitungsnachrufen und akademischen Nachrufen vorerst noch außen vor (vgl. dazu Abschn. 4), so lässt sich auch der Forschungsstand zu akademischen Nachrufen recht eindeutig in sozialstrukturanalytische und sozialkonstruktivistische Arbeiten unterteilen. Sozialstrukturell informierte Studien untersuchen dann beispielsweise die soziale Zusammensetzung der in Nachrufen erinnerten Ingenieurselite (Gispen 1994). Dem steht eine größere Auswahl von Arbeiten gegenüber, die eher sozialkonstruktivistisch argumentieren. Grundsätzlich können Nachrufe als Orte verstanden werden, in denen unter Bezug auf ein professorales Ethos akademische Subjekte hervorgebracht werden (Hamann 2016b). Mit Blick auf die Zuschreibung von Leistungen und Reputation fragen Studien zum einen, welche Marker für die Symbolisierung intellektueller Reputation herangezogen werden (Macfarlane und Chan 2014 identifizieren persönliche Eigenschaften und wissenschaftliche Leistungen) und wie sich konkrete Reputationsmarker zum anderen im Zeitverlauf und im Länder- und Disziplinenvergleich entwickeln (Hamann und Zimmer 2017 für ,Internationalität'). Nachrufe können auch zu den Standards und Erwartungen für akademische Biografien in einer Disziplin wie der Soziologie befragt werden (Hamann 2015; vgl. auch den linguistischen Beitrag zu soziologischen Nachrufen von Kresta 1996). Wie bei Zeitungsnachrufen finden sich schließlich auch Studien, die Nachrufe als Spiegel bestimmter Aspekte der akademischen Welt sehen. Sie rekonstruieren spezifische Praktiken der Konsekration akademischer Lebensläufe (Hamann 2016a), Merkmale der akademischen Wissensproduktion (von der materiellen Welt der Instrumente und Labore bis zu religiösen und politischen Loyalitäten, Echterhölter 2012) oder Indikatoren für die Dominanz der US-amerikanischen Wissenschaft (Tight 2008).

Der noch überschaubare, aber wachsende Forschungsstand verdeutlicht, dass es sich bei Nachrufen um ein fruchtbares und vielfältig nutzbares Datenmaterial handelt, das gerade auch für fachgeschichtliche Perspektiven Anknüpfungspunkte bietet. Ein Rückgriff auf Nachrufe als empirische Daten erfordert jedoch eine genauere Auseinandersetzung mit den Besonderheiten dieses Genres. Im folgenden Abschnitt will ich einige dieser Besonderheiten diskutieren.

\section{Akademische Nachrufe als Datenmaterial}

Bereits der Forschungsstand hat angedeutet, dass Nachrufe mitnichten nur das Leben von verstorbenen Wissenschaftlern dokumentieren. Es handelt sich bei dem Genre um einen Ort, an dem sich ein Forschungsfeld über sich selbst verständigt. Das kann beispielhaft an drei Aspekten illustriert werden. Erstens ist es typisch für Nachrufe, Bedingungen der Mitgliedschaft zu einem Forschungsfeld zu verhandeln. Das ist zum Beispiel der Fall wenn kommuniziert wird, warum eine Verstorbene verdientes Mitglied der betreffenden Forschungsgemeinschaft war. Ein Verstorbener mag sich beispielsweise, ,innerhalb der 
soziologischen Profession [...] neben seiner vierjährigen Konzilstätigkeit vor allem durch seine aktive und leitende Mitarbeit bei zwei Sektionen hervorgetan" haben

(Hurrelmann 1987, S. 396). Es können zweitens auch Beziehungsgeflechte innerhalb eines Kollektivs dargestellt werden, insbesondere wenn die enge Beziehung zwischen Autorin und Verstorbenem thematisiert wird. Die Erinnerung ,we met once a month for dinner and discussion for several years“ beschreibt ein Forschungsseminar nicht nur als ,small but wonderful group", sondern symbolisiert auch Vertrautheit zwischen der Autorin und dem Verstorbenem sowie eine spezifische, freundschaftliche Form des sozialen Umgangs in einem Forschungskollektiv (Bonnell 1991, S. iii). Ein weiteres sprachliches Mittel zur Markierung von Vertrautheit ist die Verwendung von Vor- oder Spitznamen der Verstorbenen - dieses Stilmittel ist aber vor allem in englischsprachigen Nachrufen zu finden (vgl. Kresta 1996, S. 134). Drittens können in Nachrufen legitime akademische Lebenspraktiken aktualisiert und reifiziert werden, wenn zum Beispiel das Arbeitsethos hochverdienter Kolleginnen und Kollegen beschrieben wird. Verstorbene können als „tireless and unselfish servant of our discipline“ (Blumer 1967, S. 103) charakterisiert werden, ein Verstorbener mag „Soziologie [als] eine Entdeckungsreise seiner selbst“ (Endreß 2002, S. 407) betrieben haben, für einen anderen war ,Sociology [...] a calling rather than just a job, something to which he devoted his life in an exceptionally wholehearted way." (Brown 1982, S. ii) Es ist zu vermuten, dass die Konsekration von hochverdienten Kollegen, bei denen „nie Ermüdung oder Resignation [...] zu verspüren“ war, die sich noch ,[b]is in die letzten Wochen und Tage hinein [...] um die Fertigstellung von Beiträgen“" (Specht 1969, S. 124) sorgten, einen disziplinierenden Druck auf jene Wissenschaftler ausübt, die (noch) einen untergeordneten Status einnehmen (vgl. dazu Hamann 2016b).

Die oben nur illustrativ skizzierten Inhalte von Nachrufen werden vor allem durch Narrative miteinander verschränkt und intelligibel gemacht (Koschorke 2012). Eine besondere Rolle spielen dabei biografische Narrative (Bamberg 2010), die aus verschiedenen, mitunter erratischen oder zufälligen Stationen eines Lebenslaufs eine kohärente und lineare Biografie konstruieren, die zielgerichtet und zwangsläufig erscheint (Bourdieu 1998). In dieser Hinsicht sind Nachrufe „Biographiegeneratoren“ (Hahn 1988, S. 93), also Arrangements, die auf die biografieförmige Abbildung von Subjektfiguren ausgerichtet sind. Element einer solchen Biographisierung kann das rhetorische Arrangement von Forschungsthemen als ein logisches und sinnvolles „Werk“ sein, beispielsweise wenn die „,hochgradige thematische Kontinuität, Konzentriertheit und Zielstrebigkeit dieses Werkes" unterstrichen wird (Klages 1963, S. 189). Auch bestimmte thematische Narrative haben eine biographisierende Wirkung. So können zum Beispiel harte Arbeit und Fleiß motivisch als übergeordnete Lebensthemen gesetzt werden. Dann ist die Rede von einem „,bedachten Menschen, harten und präzisen Arbeiter, der vorausblickend sein Leben und seine Arbeit anging und jeden Schritt genau abstimmte“ (Hurrelmann 1987, S. 395). Auf diese Weise erklären und plausibilisieren narrative Motive einzelne Stationen eines Lebenslaufs und ziehen sie zu einer einheitlichen Biografie zusammen (zu biografischen Narrativen in Nachrufen vgl. Hamann 2016a).

Das Potenzial von Nachrufen als empirischem Material besteht darin, dass sie das wissenschaftliche Personal explizieren und sichtbar machen. Die Subjektivierung ist charakterisiert durch ein relationales Verhältnis von Selbstpositionierungen (der Autoren), Fremdpositionierungen (der Verstorbenen) und der Einbeziehung eines (imaginierten) Publikums, das die vorgenommenen Positionierungen gegebenenfalls sanktionieren kann. Wie oben erwähnt können Selbstpositionierungen von Autorinnen ihre Vertrautheit mit den Verstorbenen thematisieren. Bei Fremdpositionierungen schreiben Autoren den 
Verstorbenen entweder akademische Leistungen zu, die von Preisen und Auszeichnungen über inhaltlich-fachliche Verdienste bis hin zu institutionell-administrativen Meriten reichen, oder sie schreiben Verstorbenen Charaktereigenschaften wie Fleiß, Kreativität, Humor oder Empathie zu. Selbst- und Fremdpositionierungen stehen in einem Verhältnis mit der Einbeziehung eines (imaginierten) Publikums. Ein Kollektiv wird beispielsweise angerufen wenn ein Autor erklärt, dass ,wir [...] einen Kollegen [verlieren], der viel für unsere Profession geleistet hat“ (Münch 1987, S. 625), oder dass der Verstorbene ,played a central intellectual and social role in our scholarly community" (Hargens und Gieryn 1988, S. 572). Fachgeschichtliche Studien sind gut beraten, sich dieses bereits explizit im Genre angelegte Beziehungsgeflecht von Schülern, Lehrerinnen, Kolleginnen, Weggefährten, Schulen und anderen Kollektiven bewusst zu machen.

Die starke Einbettung in personale Netzwerke sowie in darüber hinausweisende soziale Konfigurationen wie disziplinäre oder nationale Kontexte muss nicht zwingend gegen die fachgeschichtliche Verwendung von akademischen Nachrufen als empirischem Material sprechen. Im Gegenteil können aus der situationalen und kontextuellen Einbettung der Texte Vorteile erwachsen, wenn dieser Umstand in der Analyse reflektiert wird. Über dieses Potenzial ,natürlicher Daten“ verfügen ,artifizielle', also eigens für eine Studie erhobene Daten nicht (zur Unterscheidung natürlicher und artifizieller Daten vgl. Speer 2002). Im Gegenteil, mit dem Versuch, sich dem wissenschaftlichen Personal beispielsweise durch teilnehmende Beobachtung oder Interviews zu nähern, handelt man sich schnell andere Fallstricke ein: Sowohl bei Interviews als auch bei teilnehmender Beobachtung besteht die Gefahr, dass die Daten responsiv sind für Fragestellungen, Erwartungen oder auch nur die Anwesenheit der Forscherin. Das kann beispielsweise zu einem (Antwort-)Verhalten nach sozialer Erwünschtheit führen. Für Interviews gilt zusätzlich, dass es sich hierbei selbst um Biografiegeneratoren handelt, die einen eigenen Kontext für die Hervorbringung akademischer Subjektivität herstellen (vgl. Bernhard 2014). Für teilnehmende Beobachtungen ergibt sich außerdem die Schwierigkeit, dass sie im akademischen Alltag verstreute und jeweils unterschiedlich situierte Praktiken zu allgemeineren Subjektivierungsdynamiken aggregieren müssen.

Vor diesem Hintergrund besteht das analytische Potenzial von akademischen Nachrufen gerade darin, vielfältig in wissenschaftliche Praxiskontexte eingebettet und nicht eigens für die Forschungsfragen der Wissenschaftssoziologen oder -historikerinnen generiert worden zu sein. Das gilt gleichermaßen für andere in diesem Handbuch behandelte Datensorten wie beispielsweise Korrespondenzen.

\section{Akademische Nachrufe im Vergleich mit anderen Datensorten}

Aufbauend auf die obige Charakterisierung von akademischen Nachrufen kann das Genre mit ähnlichen Datensorten verglichen werden. Der explorative Vergleich erlaubt eine genauere Abgrenzung und Einordnung des Datenmaterials. Als Vergleichsfolie ziehe ich zum einen in Zeitungen veröffentlichte Nachrufe und zum anderen akademische Curricula Vitae (CVs) und das literarische Genre der Wissenschaftlerbiografie heran (zur Forschung über und mit CVs vgl. Bonzi 1992; Miller und Morgan 1993; Dietz et al. 2000, zu Biografien siehe nicht nur den Beitrag in diesem Handbuch, sondern auch Shortland und Yeo 1996; Söderqvist 2011). Bei CVs und dem literarischen Genre der Biografie handelt sich ebenfalls um Biografiegeneratoren, in denen das wissenschaftliche Personal sichtbar gemacht wird. Umso produktiver ist es, sich die Differenzen und Gemeinsamkeiten dieser 
Genres bewusst zu machen. Ich will dies hier nur anhand von drei orientierenden Fragen andeuten, und mein Fokus bleibt dabei auf akademischen Nachrufen.

Wer wird jeweils adressiert? Biografien adressieren ein relativ breites Publikum. Sie nehmen ein allgemeineres, unter Umständen populärwissenschaftliches Interesse an den von ihnen beschriebenen Lebensläufen an. Ganz ähnlich sind auch Zeitungsnachrufe für ein breiteres Publikum verfasst. Entsprechend kann weder bei Leserinnen von Zeitungen noch von (populärwissenschaftlichen) Sachbüchern eine intime Kenntnis disziplinärer, geschweige denn subdisziplinärer Besonderheiten vorausgesetzt werden. Ganz anders richten sich $\mathrm{CVs}$ an ein enger definiertes Publikum. In der Regel sollen sie potenzielle Arbeitgeber über bisherige Stationen und Leistungen unterrichten. Ein Spannungsverhältnis kann zwischen dem hohen Formalisierungsgrad von CVs und der Notwendigkeit bestehen, sie dennoch an die (antizipierten) Erwartungen unterschiedlicher Arbeitgeber anzupassen. Wieder anders ausgerichtet sind akademische Nachrufe. Sie adressieren ein spezifisches Publikum, nämlich die Fachgemeinschaft, die um ihr Mitglied trauert. Das wird allein am Veröffentlichungsort der Fachzeitschrift deutlich. Weil akademische Nachrufe auf die Rezeption durch eine Fachgemeinschaft ausgerichtet sind, schlagen sich fachspezifische Aspekte in ihnen deutlich nieder. Biografien und Zeitungsnachrufe stellen Wissenschaftlerinnen als Personen des öffentlichen Interesses dar, während CVs zu stark formalisiert sind, um disziplinäre Differenzen zu reflektieren. Aufgrund ihrer spezifischen Publikumsorientierung sind akademische Nachrufe daher besonders für fachgeschichtliche Fragestellungen geeignet.

Wer ist der Autor? Biografien können von Schülerinnen oder Familienmitgliedern der biografisierten Person verfasst werden, in der Regel sind es aber Feuilletonisten, Historikerinnen oder professionelle Biografen. Bei CVs ist davon auszugehen, dass sie grundsätzlich von der Person verfasst wurden, deren Lebenslauf dargestellt wird. Die beiden Autorinstanzen unterscheiden sich in ihrer Vertrautheit mit der dokumentierten Biografie: Biografen, Feuilletonisten und Historikerinnen müssen diese Vertrautheit durch oft langwierige Recherchen in Archiven und bei Familienmitgliedern erst herstellen. Demgegenüber werden sich schwerlich Autorinnen finden, die vertrauter mit einem darzustellenden Leben sind als jene, die ihr eigenes CV verfassen. Diese Beobachtungen stellen einen analytisch fruchtbaren Kontrast zu Nachrufen her: Während Zeitungsnachrufe nicht zwingend, aber oft von vertrauten Wegbegleitern geschrieben werden - hier können auch Journalistinnen die Autorschaft übernehmen -, sind akademische Nachrufe in aller Regel von Schülern, Freundinnen oder Kollegen verfasst. Sie verwenden, darauf habe ich bereits im vorhergehenden Abschnitt hingewiesen, einen ganz wesentlichen Teil des Textes darauf, ihre Vertrautheit mit der verstorbenen Person glaubhaft zu machen. Autorinnen akademischer Nachrufe fällen Urteile über verdiente Mitglieder, und diese Urteile werden aufmerksam verfolgt von einer Fachgemeinschaft, die gegebenenfalls aus weiteren Schülern, Freundinnen und Kollegen der Verstorbenen besteht. Der Vergleich mit anderen Biografiegeneratoren erinnert die Fachgeschichte also daran, dass Autorinnen akademischer Nachrufe zum einen als enge Vertraute verdienter Kollegen fungieren, und zum anderen als die „ordnungsgemäß beauftragten Sprecher“, die „[d] as letzte Urteil“" sprechen, das die Gruppe über eines ihrer Mitglieder fällt (Bourdieu 1992, S. 379). Diese soziale Dimension von akademischen Nachrufen kann nicht zuletzt in soziologiegeschichtlichen Studien produktiv gewendet werden.

Aus den Fragen nach den Publika und den Autorinnen der verschiedenen Genres folgt bereits die Anschlussfrage, wie das wissenschaftliche Personal jeweils dargestellt wird. Das literarische Genre der Biografie verknüpft in der Regel einen Lebenslauf mit allgemeineren 
Kontexten - entweder um durch den Lebenslauf einen allgemeineren Kontext, beispielsweise die Geschichte einer Disziplin oder eine historischen Epoche zu erhellen, oder weil der spezifische Lebenslauf über einen allgemeineren Kontext erklärt werden soll. Bei CVs geht es dagegen gerade darum, klar definierte Stationen aus ihren Kontexten herauszuschälen. Das geht so weit, dass selbst die chronologische Struktur eines Lebenslaufs aufgebrochen wird, um anderen Kategorien Raum zu geben - in der Regel sind dies dann Leistungsmarker wie Publikationen, Lehrerfahrung oder Auslandsaufenthalte. Die gesellschaftlichen oder disziplinären Kontexte, in die diese Stationen und Leistungen eingebettet waren, spielen hier keine Rolle. Für die fachgeschichtliche Analyse ergeben sich daher sehr spezifische Nutzungsmöglichkeiten: Die Darstellung des wissenschaftlichen Personals in Biografien wird nicht selten von feldexternen Autoren vorgenommen und folgt daher auch oft anderen Maßstäben der Relevanz und der Dramaturgie. CVs dagegen stellen das wissenschaftliche Personal so stark dekontextualisiert dar, dass einerseits wenige Einblicke hinsichtlich jener Kategorien möglich sind, die die Fachgeschichte üblicherweise interessieren (bspw. die Geschichte von Institutionen, Schulen, Fachgesellschaften oder Fachzeitschriften). Andererseits eröffnet gerade der hohe Standardisierungsgrad von CVs Möglichkeiten breiter angelegter, historischer oder ländervergleichender Fachgeschichten (vgl. die aus dem ERC-geförderten Projekt DISCONEX (Warwick/Paris) hervorgehenden Arbeiten, z. B. Angermuller 2016; Hamann et al. 2017).

Die Darstellung des wissenschaftlichen Personals in Nachrufen folgt eigenen Regeln. Nachrufe in Zeitungen stellen Lebensläufe generell in einem positiven Licht dar (Bonsu 2007, S. 207-209; Long 1987, S. 988-989). Dennoch gibt es durchaus Raum für negative Aspekte wie ungünstige Umstände oder vergebliche Hoffnungen (Fowler 2007, S. 63). Im Vergleich dazu besteht in akademischen Nachrufen eine stärkere Tendenz, Rückschläge oder externe Zwänge zu verschleiern (Macfarlane und Chan 2014; Tight 2008). Die vielgestaltigen Krisen, die Wissenschaftlerinnen in ihren Leben durchlaufen, können in der Regel nur durch Heranziehung anderer Datensorten sichtbar gemacht werden. Die Triangulation von Nachrufen mit beispielsweise Interviews oder autobiografischen Texten erhält hier eine besondere Relevanz. Erst die Kontrastierung mit anderen Materialien macht deutlich, in welchem Umfang Krisen, Zwänge und Rückschläge in Nachrufen entweder nicht erwähnt oder im Rahmen einer Heldengeschichte zu einem überwundenen Hindernis umgedeutet werden. An anderer Stelle habe ich diese Auslassungen und Umdeutungen als Ergebnis einer durch informelle Konsekrationsregeln des Genres ausgeübten Zensur interpretiert (Hamann 2016a). Für die Soziologiegeschichte ist es freilich besonders bemerkenswert, dass Biografien gerade auch in soziologischen Nachrufen weitgehend unsoziologisch erzählt werden: Soziale Einflüsse werden invisibilisiert, das Lebenswerk erscheint als kreative Eigenleistung des Verstorbenen. 2

Zwar sollten sich fachgeschichtliche Studien der im Genre üblichen selektiven Selbst - und Fremddarstellung bewusst sein, um eine bloße Nacherzählung von akademischen Heldengeschichten zu vermeiden (vgl. zu einer ähnlichen Problematik den Beitrag zu Korrespondenzen in diesem Handbuch). Auf der anderen Seite können gerade die diskursiven Zensur- und Selektionsmechanismen analytisch genutzt werden, explizieren sie doch die nicht immer offen liegenden Ethiken und Regeln, entlang derer die Fachgeschichte die symbolische Ordnung eines Feldes rekonstruieren kann (siehe dazu Hamann 2016b). 


\section{Fazit}

Akademische Nachrufe stellen fruchtbares Datenmaterial für soziologie- und andere fachgeschichtliche Fragestellungen dar. Ich habe in diesem Beitrag vor allem auf ihre spezifischen Produktions- und Rezeptionskontexte abgestellt. Die soziale Beziehung zwischen Autorin und Verstorbenen, der jeweilige disziplinäre Kontext eines Nachrufs und die durch die veröffentlichende Fachzeitschrift angezeigte subdisziplinäre Fachgemeinschaft müssen ebenso analytisch reflektiert werden wie die spezielle Funktion von Nachrufen, wissenschaftliche Biografien und Lebensleistungen nicht objektiv, sondern konsekrierend darzustellen. Der fachgeschichtliche Fokus liegt dann weniger darauf, Nachrufe als quasi-positivistische Dokumentation eines tatsächlich auf diese Weise gelebten Lebens in realistisch abgebildeten Kontexten nachzuvollziehen. Von größerem Interesse sind die Narrative, Zuschreibungen, Selbst- und Fremdpositionierungen und Repräsentationen, die bei der feierlichen Ehrung verstorbener Schüler, Kolleginnen und Weggefährten im Material mobilisiert werden. Sie machen sichtbar, wie wissenschaftliche Personen und Lebensleistungen abgebildet und bewertet werden und geben so den Blick frei auf ansonsten weitgehend beschwiegene Regeln der anerkannten akademischen Lebensführung. Gelingt diese analytische Reflexion, eröffnet sich für die Soziologiegeschichte ein noch weitgehend unbestelltes Feld.

\section{Fußnoten}

1. Der Text wechselt zwischen generischem Maskulinum und Femininum. Dies soll aber nicht darüber hinwegtäuschen, dass Nachrufe in Fachzeitschriften ein Genre sind, das vornehmlich von Männern für Männer geschrieben wird.

2. Für diesen Hinweis danke ich Stephan Moebius.

\section{Literatur}

Angermuller, Johannes. 2016. Akademische Subjektivierung. Was Statuskategorien über wissenschaftliche Karrieren in Frankreich im Vergleich zu den USA, Großbritannien und Deutschland aussagen. In Macht in Wissenschaft und Gesellschaft. Diskurs- und feldanalytische Perspektiven, Hrsg. Julian von Hamann, Jens Maeße, Vincent Gengnagel und Alexander Hirschfeld, 25-54. Wiesbaden: Springer.

Bamberg, Michael G. W. 2010. Who am I? Narration and its contribution to self and identity. Theory \& Psychology 21(1): 1-22.

Bernhard, Stefan. 2014. Identitätskonstruktionen in narrativen Interviews. Ein Operationalisierungsvorschlag im Anschluss an die relationale Netzwerktheorie. Forum Qualitative Sozialforschung 15(3), Art 1.

Blumer, Herbert. 1967. Ernest Watson Burgess, 1886-1966. The American Sociologist 2(2): 103-104.

Bonnell, Victoria. 1991. In memory of Reinhard Bendix. Berkeley Journal of Sociology 36(1991): i-v.

Bonsu, Samuel K. 2007. The presentation of dead selves in everyday life: Obituaries and impression management. Symbolic Interaction 30(2): 199-219.

Bonzi, Susan. 1992. Trends in research productivity among senior faculty. Information Processing \& Management 1992(28): 111-120.

Bourdieu, Pierre. 1992. Homo academicus. Frankfurt a. M.: Suhrkamp.

Bourdieu, Pierre. 1998. Die biographische Illusion. In Praktische Vernunft. Zur Theorie des Handelns, Hrsg. Pierre von Bourdieu, 75-90. Frankfurt a. M.: Suhrkamp. 
Bourdieu, Pierre. 2004. Science of science and reflexivity. Cambridge: Polity Press.

Brown, Richard. 1982. Philip Abrams 1933-1981. Sociology 16(1): i-iii.

Bytheway, Bill, und Julia Johnson. 1996. Valuing lives? Obituaries and the life course. Mortality 1(2): 219-234.

Dietz, James S., Ivan Chompalov, Barry Bozeman, Eliesh O`Neil Lane, und Jongwon Park. 2000. Using the curriculum vita to study the career paths of scientists and engineers: An exploratory assessment. Scientometrics 49(3): 419-422.

Echterhölter, Anna. 2012. Schattengefechte. Genealogische Praktiken in Nachrufen auf Naturwissenschaftler (1710-1860). Göttingen: Wallstein Verlag.

Eid, Mushira. 2002. The world of obituaries: Gender across cultures and over time. Detroit: Wayne State University Press.

Emihovich, Catherine. 1995. Distancing passion: Narratives in social science. International Journal of Qualitative Studies in Education 8(1): 37-48.

Endreß, Martin. 2002. Nachruf auf Heinrich Popitz (1925-2002). Berliner Journal für Soziologie 3(2002): 405-409.

Etzemüller, Thomas. 2013. Der ,Vf.‘ als Subjektform. Wie wird man zum ,Wissenschaftler und (wie) lässt sich das beobachten? In Selbst-Bildungen. Soziale und kulturelle Praktiken der Subjektivierung, Hrsg. Thomas von Alkemeyer, Gunilla Budde und Dagmar Freist, 175-196. Bielefeld: transcript.

Foucault, Michel. 1977. What is an author? In Language, counter-memory, practice: Selected essays and interviews, Hrsg. Michel von Foucault, 113-138. New York: Cornell University Press.

Foucault, Michel. 1991. Die Ordnung des Diskurses. Frankfurt a. M.: Fischer.

Fowler, Bridget. 2005a. Collective memory and forgetting: Components for a study of obituaries. Theory Culture \& Society 22(6): 53-72.

Fowler, Bridget. 2005b. Mapping the obituary: Notes towards a bourdieusian interpretation. The Sociological Review 52(2): 148-171.

Fowler, Bridget. 2007. The obituary as a collective memory. New York: Routledge.

Fowler, Bridget, und Esperança Bielsa. 2007. The lives we choose to remember: A quantitative analysis of newspaper obituaries. The Sociological Review 55(2): 203-226.

Gispen, Kees. 1994. Die deutsche Ingenieurelite, 1840-1930: eine Analyse der Nachrufe. In Ingenieure in Deutschland, 1770-1990, Hrsg. Peter von Lundgreen und Andre Grelon, 221-241. Frankfurt a. M.: Campus.

Gusfield, Joseph. 1976. The literary rhetoric of science: Comedy and pathos in drinking driver research. American Sociological Review 1976(41): 16-34.

Hahn, Alois. 1988. Biographie und Lebenslauf. In Vom Ende des Individuums zur Individualität ohne Ende, Hrsg. Hanns-Georg von Brose und Bruno Hildenbrand, 91105. Opladen: Leske + Budrich.

Hamann, Julian. 2015. Posthumous (E)valuation. Research biographies in US sociology, as reflected in academic obituaries. Timelines - Newsletter of the ASA Section History of Sociology 2015(24): 10-13.

Hamann, Julian. 2016a. ,Let us salute one of our kind'. How academic obituaries consecrate research biographies. Poetics 56(2016): 1-14.

Hamann, Julian. 2016b. Wie entstehen wissenschaftliche Subjekte? Zum professoralen Ethos akademischer Lebenspraxis. In Macht in Wissenschaft und Gesellschaft. Diskurs- und feldanalytische Perspektiven, Hrsg. Julian von Hamann, Jens Maeße, Vincent Gengnagel und Alexander Hirschfeld, 83-111. Wiesbaden: Springer.

Hamann, Julian, und Zimmer Lena M. 2017. The internationality imperative in academia. The ascend of internationality as an academic virtue. Manuskript im Review.

Hamann, Julian, Jens Maeße, Ronny Scholz, und Johannes Angermuller. 2017. From text to the social: Studying the dispositif between discourse analysis, quantitative context 
mapping, and interpretative theorizing. In Quantifying approaches to discourse for social scientists, Hrsg. Ronny von Scholz, S. i. E. New York: Palgrave Macmillan.

Hargens, Lowell L., und Thomas F. Gieryn. 1988. Nicholas C. Mullins (1939-88). Social Studies of Science 18(3): 572-573.

Hurrelmann, Klaus. 1987. Hans Haferkamp (15.09.1939-14.07.1987). Zeitschrift für Soziologie 16(5): 395-398.

Hyland, Ken. 2004. Disciplinary discourses. Social interactions in academic writing. Ann Arbor: University of Michigan Press.

Klages, Helmut. 1963. Karl Valentin Müller. Soziale Welt 14(2): 189-190.

Kohli, Martin. 1981. ,Von uns selber schweigen wir." Wissenschaftsgeschichte aus Lebensgeschichten. In Geschichte der Soziologie. Studien zur kognitiven, sozialen und historischen Identität einer Disziplin, Hrsg. Wolf von Lepenies, 4 Bde, 428-465. Frankfurt a. M.: Suhrkamp.

Koschorke, Albrecht. 2012. Wahrheit und Erfindung: Grundzüge einer Allgemeinen Erzähltheorie. Frankfurt/M.: Fischer.

Kresta, Ronald. 1996. ,Nachrufe' in englischen und deutschen Fachzeitschriften in der Soziologie. Fachsprache 1996(18): 118-137.

Long, Gary L. 1987. Organizations and identity: Obituaries 1856-1972. Social Forces 65(4): 964-1001.

Macfarlane, Bruce, und Roy Y. Chan. 2014. The last judgement: Exploring intellectual leadership in higher education through academic obituaries. Studies in Higher Education 39(2): 294-306.

Merton, Robert K. 1973. The normative structure of science. In The sociology of science, Hrsg. Robert K. von Merton, 267-278. Chicago: University of Chicago Press.

Miller, Nod, und David Morgan. 1993. Called to account: The CV as an autobiographical practice. Sociology 27(1): 133-143.

Münch, Richard. 1987. In memoriam Hans Haferkamp (15.09.1939-14.07.1987). Kölner Zeitschrift für Soziologie und Sozialpsychologie 1987(39): 625-628.

Rodler, Christa, Erich Kirchler, und Erik Hölzl. 2001. Gender stereotypes of leaders: An analysis of the contents of obituaries from 1974 to 1998. Sex Roles 45(11-12): 827-843.

Shortland, Michael, und Richard Yeo, Hrsg. 1996. Telling lives in science: Essays on scientific biography. Cambridge: Cambridge University Press.

Söderqvist, Thomas. 2011. The seven sisters: Subgenres of bioi of contemporary life scientists. Journal of the History of Biology 2011(44): 633-650.

Specht, Karl G. 1969. Leopold v. Wiese. Soziale Welt 20(1): 124-125.

Speer, Susan A. 2002. ,Natural' and ,contrived' data: A sustainable distinction? Discourse Studies 4(4): 511-525.

Taussig, Doron. 2016. Your story is our story: Collective memory in obituaries of US military veterans. Memory Studies (online first).

Tight, Malcom. 2008. Dead academics: What can we learn about academic work and life from obituaries? London Review of Education 6(2): 125-135.

Weber, Max. 1922. Wissenschaft als Beruf. In Gesammelte Aufsätze zur Wissenschaftslehre, Hrsg. Weber von Max, 524-555. Tübingen: J.C.B. Mohr. zuerst 1919. 